\title{
Green revolution in chemistry by microwave assisted synthesis: A review
}

\section{Savita Belwal}

Department of Chemical Engineering, CVSR College of Engineering, Hyderabad (A. P.)

\section{Email address:}

savita_gopal@rediffmail.com

\section{To cite this article:}

Savita Belwal. Green Revolution in Chemistry by Microwave Assisted Synthesis: A Review. Modern Chemistry. Vol. 1, No. 3, 2013, pp. 22-25. doi: 10.11648/j.mc.20130103.11

\begin{abstract}
Green chemistry efficiently utilizes raw materials (preferably renewable), eliminates waste, and avoids the use of toxic or hazardous reagents and solvents in the manufacture and application of chemical products. Microwave assisted technique opens up new opportunities to the synthetic chemist in the form of new reactions that are not feasible using conventional heating and serve a flexible platform for chemical reactions. Over the past five years there has been a dramatic uptake in the use of microwaves as an energy source to promote synthetic transformations. Microwave-assisted synthesis (MAOS) is clearly a method by which the laboratory chemist can achieve goals in a fraction of the time as compared to traditional conductive heating methods. Reaction times in the best cases have been reduced from hours or days to minutes. The basic mechanisms observed in microwave assisted synthesis are dipolar polarization and conduction. The technique offers a simple, clean, fast, efficient, and economic way for the synthesis of a large number of molecules, providing the momentum for many chemists to switch from traditional heating methods to microwave assisted chemistry. In the present article an attempt was made to focus on what is microwave assisted synthesis, how is it generated and what importance may it have.
\end{abstract}

Keywords: Microwave Radiation, Electromagnetic Spectrum, Green Chemistry

\section{Introduction}

Microwave irradiation (MWI) has become an established tool in chemical synthesis, because of the rate enhancements, higher yields, and often, improved selectivity, with respect to the conventional reaction conditions. ${ }^{1,2}$ In recent years, solvent -free reactions using either organic or inorganic solid supports have received increasing attention. ${ }^{3,4}$ There are several advantages to performing synthesis in dry media: (i) short reaction times, (ii) increased safety, (iii) economic advantages due to the absence of solvent. In addition, solvent free MWI processes are also clean and efficient.

Synthesis of new chemical entities is a major bottleneck in drug discovery. Conventional methods for various chemical syntheses are very well documented and practiced $^{5}$. The methods for synthesis of chemical compounds have continuously been modified through the decades. Now-a-day'-s the microwave technique is considered as an important approach toward green chemistry, because this technique is more environmentally friendly. This technology is still under-used in the laboratory and has the potential to have a large impact on the fields of screening, combinatorial chemistry, medicinal chemistry and drug development. Due to its ability to couple directly with the reaction molecule and by-passing thermal conductivity leading to a rapid rise in the temperature, microwave irradiation has been used to improve many organic syntheses.

A microwave is a form of electromagnetic energy, which falls at the lower end of the electromagnetic spectrum and is defined in a measurement of frequency as 300 to 300,000 , Megahertz, corresponding to wavelengths of $1 \mathrm{~cm}$ to $1 \mathrm{~m}$. The microwave region of the electromagnetic spectrum lies between infrared and radio frequencies ${ }^{6,7}$. The difference between microwave energy and other forms of radiation, such as $\mathrm{X}$ - and $\gamma$-rays, is that microwave energy is nonionizing and therefore does not alter the molecular structure of the compounds being heated - it provides only thermal activation. The heating effect utilized in microwave assisted transformations is mainly due to dielectric polarization. When a molecule is irradiated with microwaves, it aligns itself with the applied field. The rapidly changing electric field $(2.45 \times 109 \mathrm{~Hz})$ affects the molecule and consequently 
the molecule continually attempts to align itself with the changing field thus energy is absorbed. The ability of a material to convert electromagnetic energy into thermal energy is dependent on the dielectric constant. The larger the dielectric constant the greater is the coupling with microwaves. Thus, solvents such as water, methanol, DMF, ethyl acetate, acetone, acetic acid, etc. are all heated rapidly when irradiated with microwaves. However, solvents with low dielectric constants such as hexane, toluene, carbon tetrachloride, etc. do not couple and therefore do not heat that rapidly under microwave irradiation. Microwave heating has thus been found to be a very convenient thermal source not only in the kitchen but also in a chemical laboratory ${ }^{8,9}$. Chemists have explored the possibility of the application of a conventional microwave oven to carry out chemical reactions. However, the advantages of using microwave dielectric heating for performing organic transformations ${ }^{10}$ have only emerged since the mid- 1980s. This technology opens up new opportunities to the synthetic chemist, in the form of new reactions that are not possible using conventional heating.

The concepts of atom economy and energy factors have eventually become a guiding principle ${ }^{11}$ of Green chemistry. These are embodied in the " 12 Principles of Green chemistry" which are as follows:

- Prevention of waste

- Atom Economy

- Less Hazardous Chemical Syntheses

- Design Safer Chemicals

- Safer Solvents and Auxiliaries

- Design for Energy Efficiency

- Use Renewable Feedstocks

- Reduce Derivatives

- Catalysis

- Design for Degradation

- Real-time Analysis for Pollution Prevention

- Inherently Safer Chemistry for Accident Prevention

\section{Microwave Heating and Principle}

The basic principle behind the heating in a microwave oven resides to the interaction of the charged particles of the reaction material with electromagnetic waves of particular frequency. The phenomena of producing heat by electromagnetic irradiation are either by collision or by conduction, sometimes even by both ${ }^{12,13}$.

All the wave energy changes its polarity from positive to negative with each cycle of the wave. This causes a rapid orientation and reorientation of molecules, resulting to heating by collision ${ }^{14,15}$. If the charged particles of the material are free to travel through the material (e.g. electron in a sample of carbon), a current will be induced which will travel in phase with the field. If the charged particles are bound within regions of the material, the electric field component will cause them to move until the opposing force balances the electric force ${ }^{16-19}$.

\section{Microwave for Synthesis}

In nineteenth century the Bunsen burner was later superseded by the isomantle, oil bath or hot plate as a source of applying heat to a chemical reaction. In the past few years, heating chemical reactions by microwave energy has been an increasingly popular theme in the scientific community. Since the first published reports on the use of microwave irradiation to carry out organic chemical transformations by the groups of Gedye ${ }^{20}$, more than 5000 articles have been published in this fast moving and exciting field, today generally referred to as microwaveassisted organic synthesis ${ }^{21-23}$. In the early days of microwave synthesis, experiments were typically carried out in sealed Teflon or glass vessels in a domestic household microwave oven without any temperature or pressure measurements. Kitchen microwave ovens are not designed for the rigors of laboratory usage: acids and solvents corrode the interiors quickly and there are no safety controls. The results were often violent explosions due to the rapid uncontrolled heating of organic solvents under closed vessel conditions.

In the 1990s several groups started to experiment with solvent-free microwave chemistry (so-called dry-media reactions), which eliminated the danger of explosions ${ }^{24}$. Here, the reagents were pre adsorbed onto either a more or less microwave transparent inorganic support (i.e., silica, alumina or clay) or a strongly absorbing one (i.e., graphite), that additionally may have been doped with a catalyst or reagent. In many of the published examples, microwave heating has been shown to dramatically reduce reaction times, increase product yields and improve product purities by reducing unwanted side reactions compared to conventional heating methods. The advantages of this enabling technology have, more recently, also been exploited in the context of multistep total synthesis ${ }^{25}$ and medicinal chemistry/drug discovery ${ }^{26}$, and have additionally penetrated related fields such as polymer synthesis $^{27}$, material sciences ${ }^{28}$, nanotechnology ${ }^{29}$ and biochemical processes ${ }^{30}$. The use of microwave irradiation in chemistry has thus become such a popular technique in the scientific community that it might be assumed that, in a few years, most chemists will probably use microwave energy to heat chemical reactions on a laboratory scale. The statement that, in principle, any chemical reaction that requires heat can be performed under microwave conditions has today been generally accepted as a fact by the scientific community.

\section{Reaction Medium (In Presence of Solvent)}

The reactions are carried out in a solvent medium ${ }^{31}$ or on the solid support (in which no solvent is used). For a reaction in solvent medium, the solvent must have a dipole moment so as to absorb microwaves and a boiling point at least 20-30 higher than the reaction temperature. In this 
respect N,N-dimethylformamide (DMF), is an excellent solvent used in microwave oven with high b.p. $\left(160^{\circ} \mathrm{C}\right)$ and high dielectric constant $(\varepsilon=36.7)$. Other solvents used are: formamide (b.p. $216^{\circ} \mathrm{C}, \varepsilon=111$ ), methanol (b.p. $65^{\circ} \mathrm{C}, \varepsilon=$ 32.7 ), 1,2-dichlorobenzene (b.p. $83^{\circ} \mathrm{C}$ ), dioxane (b.p. $101^{\circ} \mathrm{C}$ ). The presence of salt in polar solvents can frequently enhance, microwave coupling.

Hydrocarbon solvents such as hexane $(\varepsilon=1.9)$, toluene ( $\varepsilon$ $=1.9$ ), because of less dipole moment are unsuitable as they absorb microwave radiation poorly but addition of small amounts of alcohol or water can lead to a dramatic coupling effect ${ }^{32}$.

\section{Chemical Reactions without Solvent (Over Solid Supports)}

Recent years have witnessed the importance of microwaves in mediating reactions ${ }^{33}$ because of the advantages over with classical chemistry in terms of shorter reaction times, minimum waste, generally higher yields, possibility of carrying the reactions in the absence of solvents and in safe conditions. In view of this, more interest has now been focused on dry media synthesis, involving the coupling of MWI with solid supported reagents.

Solid support method was initially described by Keinan and Mazur ${ }^{34}$. Solvent free techniques represent a clean economical, efficient and safe procedure which can lead to substantial saving in time, money and products. The expeditious and solvent-free approach involves the exposure of neat reactants to microwave irradiation in conjugation with the use of supported reagents or catalysts, which are primarily of mineral origin. Mineral oxides are poor conductor of heat but they absorb microwave radiation very effectively with significant improvement in temperature, homogeneity and heating rates which enables faster reactions and less degradation of final products. Depending on the type of organic reactions aluminas, silicas, clays, zeolites or envirocates EPZG and EPZ 10 are selected as acidic or basic supports ${ }^{35,36}$. The recyclability of various solid supports from the reaction medium is the most interesting feature as it renders these processes truly eco-friendly, reduce the pollution at source and make the process to fall in the domain of important green chemical theme.

\section{Microwave Heating in Drug Discovery}

During the past fifteen years there is a tremendous growth and efforts to prepare organic ${ }^{37}$, inorganic and organometallic compounds via green chemical approach.

Introduction of Green technology in drug discovery ${ }^{38,39}$ can help streamline process improvements in the R\&D field. Chart shown below depicts R\&D philosophy in harmony with the principles of Green Chemistry

\begin{tabular}{|c|c|c|}
\hline & Environmentally Thinking & Economically Thinking \\
\hline Atom Economy & Minimal by-product formation & More from less - incorporate total value of materials \\
\hline Solvent Reduction & Less solvent waste & Higher throughput, less energy \\
\hline Reagent Optimization & Catalytic, low stoichiometry, recyclable reagents minimize usage & Higher efficiency - higher selectivities \\
\hline Convergency & due to increased process efficiency & Higher efficiency - fewer operations \\
\hline Energy Reduction & from power generation, transport and use & $\begin{array}{l}\text { Reduced energy reflects increased efficiency, shorter } \\
\text { process, mild conditions }\end{array}$ \\
\hline In-situ Analysis & Reduced possibility for exposure or release to the environment & $\begin{array}{l}\text { Real-time data increases throughput and process } \\
\text { efficiency, fewer reworks }\end{array}$ \\
\hline Safety & $\begin{array}{l}\text { Non-hazardous materials reduce risk of exposure, release, } \\
\text { explosions \&fires }\end{array}$ & $\begin{array}{l}\text { Worker safety and reduced down time Reduced time on } \\
\text { special control measures }\end{array}$ \\
\hline
\end{tabular}

Microwave assisted green synthesis is a very good technique in the field of green chemistry by offering a flexible platform for many named organic reactions. This technology is still under-used in the laboratory and has the potential to have a large impact on the fields of screening, combinatorial chemistry, medicinal chemistry and drug development. The compiled review gives an idea on MW green chemistry, its principle, its assembly and enlists some research work which will be beneficial for promising scientists.

\section{Conclusion and Perspectives}

The term "green chemistry" is defined as "the invention, design and application of chemical products and processes to reduce or eliminate the use and generation of hazardous substances. Green chemistry can diminish the need for other approaches to environmental protection. Ideally, the application of green chemistry principles and practices renders regulation, control, clean-up, and remediation of the environment. Thus its benefits can be expressed in terms of economic impact.

Current microwave reactors are able to translate small scale microwave chemistry from milligram or gram scale to multi-kilogram scale using batch or continuous-flow processing. However, many of the benefits of small scale microwave chemistry are lost when the processes performed in larger batch reactors.

The second disadvantage is equipment cost. Although the cost of microwave reactors have dramatically reduced in recent years, the current price range is still many times higher than that of conventional heating equipment, 
considerably limiting the uptake of this valuable technology.

Drug discovery is a challenging and demanding symphony, no one technique alone can whistle it. It is required to take a whole orchestra of strategies and techniques to play it well. In combination there is strength.

Microwave chemistry has already been constantly combined with other enabling technologies and strategies such as multi-component reactions, solid-phase organic synthesis, or combinatorial chemistry. The combination of multidisciplinary approach with microwave heating encourage scientists to initiate new and unexplored areas of complex pharmaceutical systems. To date, microwave irradiation still needs to find promising and tempting combinations with more techniques to satisfy the

increased synthetic demands in industry and academia.

All in all, microwave heating is undoubtedly a bonanza for organic and medicinal chemistry researchers. This enabling technique has changed from the 'last sort' in early days to the 'first choice' nowadays for carrying out synthetic transformations requiring heat.

In the future, with lower costs, microwave synthesizers will become an integral part of and a standard technology in most synthetic laboratories and will continually make valuable impact on both organic synthesis and drug discovery.

\section{References}

[1] Lidstrom P, Tierney J, Wathey B \& Westman, J Tetrahedron, 57 (2001) 9225.

[2] Kirschning A, Sonnenschein H \& Wittenberg R, Angew. Chem Int Ed, 73 ( 2001) 193.

[3] Varma R S, Pure Appl Chem, 73 (2001) 1309.

[4] Diddams P \& Butters M, Solid Supports and Catalysts in Organic Synthesis, Smith K, Ed, Ellis Harwood and PTR Prentice Hall: New York and London (1992) Chapters 1, 3 and 5 .

[5] Anastas P T \& Warner J C, Green Chemistry, Theory and Practice, Oxford University Press: Oxford (1998).

[6] Lancaster M, Green Chemistry: An Introductory Text, The Royal Society of Chemistry: London (2002).

[7] Nagariya A K, Meena, Kiran A K, Yadav A K, Niranjan U S, Pathak A K , Singh B \& Rao M M, J Pharm Res, 3 (3) (2010) 575 .

[8] Kappe C O \& Dallinger D, Nat Rev Drug Discovery, 5 (2006) 51.

[9] Heravi M M, Ajami D, Mojtahedi M M \& Ghassemzadeh M, Tetrahedron Lett, 40 (1999) 561.

[10] Abramovich R A, Org Prep Proceed Int, 23 (1991) 638.

[11] Anastas P T \& Farris C A, ACS Symp, American Chemical Society, Washington DC, 577 (1994).

[12] Adam D, Nature, 421 (2003) 571.
[13] Blackwell H E, Org Biomol Chem, 1, (2003) 1251.

[14] Sharma S V, Rama-sarma G V S \& Suresh B, Indian J Pham Scien, 64 (2002) 337.

[15] Johansson H, Am Lab, 33 (2001) 28.

[16] Bradley D, Modern Drug Discovery, 4 (2001) 32.

[17] Larhed M, Hall berg A, Drug Discovery Today, 6 (2001) 406.

[18] Wathey B, Tierney J, Lidrom P \& Westman, J Drug Discovery Today, 7 (2002) 373.

[19] Dzieraba C D \& Combs A P, Annual reports in medicinal chemistry, academic press, 37 (2002) 247.

[20] Gedye R, Smith F, Westaway K \& Ali H, Tetrahedron Lett, 27(1986) 279.

[21] Lidstrom P, Tierney J \& Wathey B, Tetrahedron, 57 (2001) 7764.

[22] Kidwai M, Pure Appl Chem, 78 (11) (2006) 1983.

[23] Ravichandran S \& Jeyachandramani N, Int J Chem Sci, 5(3) (2008) 1258.

[24] Varma R S, Tetrahedron, 58 (2002) 1235.

[25] Artman D D \& Williams R M, J Amer Chem Soc, 129 (2007) 6336.

[26] Ravichandran S, Subramani K, Kumar R Arun, Int J Chem Sci, 6 (2008) 1800.

[27] Zhang C, Liao L \& Gong S, Green Chemistry, 9 (2007) 303.

[28] Sinnwell S \& Ritter H, Aus J Chem, 10 (2007) 729.

[29] Tsuji M, Hashimoto M, Nishizawa Y, Kubokawa M \& Tsuji T, Chemistry - A European Journal, 11 (2005) 440.

[30] Collins J M \& Leadbeater N E, Organic and Biomolecular Chemistry, 5 (2007) 1141.

[31] Hayes B L \& Mathews N C, "Microwaves Synthesis, Chemistry at the speed of light", CEM Publishing, 2002.

[32] Huibers G, Folk J A, Patel S R \& Marcus C M, Phys Rev lett, 83(24) (1999) 5090.

[33] Lidstrom P, Tierney J, Wathey B \& Westman J, Tetrahedron, 57 (2001) 9225.

[34] Cohen Z, Keinan E, Mazur Y \& Varkony T H, J Org Chem, 40 (1975) 2141.

[35] Smith K, Solid Support and Catalysis in Organic Synthesis, Ed Ellis Horwood, PTR Prentice Hall: Chichester, 1992.

[36] Clark J H, Catalysis of organic reaction by supported inorganic reagents, Ed VCH: Weinheim, 1994.

[37] Kidwai M, Kumar R, Srivastava A \& Gupta H P, Bioorg Chem, 26 (1998) 289.

[38] Matloobi M \& Kappe C O, J Combinato Chem, 9(2) (2007) 275.

[39] Kappe C O, Chem Soc Rev, 37(6) (2008) 1127. 